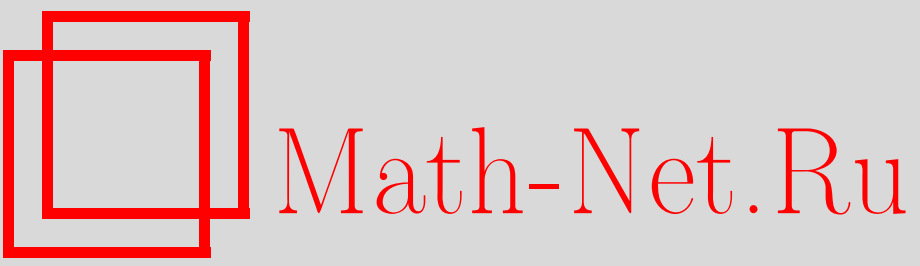

В. И. Афанасьев, Принцип инвариантности для критического ветвящегося процесса в случайной среде, достигающего высокого уровня, Теория вероятн. и ее примен., 2009, том 54, выпуск 1, 3-17

DOI: https://doi.org/10.4213/tvp2496

Использование Общероссийского математического портала Math-Net.Ru подразумевает, что вы прочитали и согласны с пользовательским соглашением

http: //www . mathnet.ru/rus/agreement

Параметры загрузки:

IP : 54.224 .135 .184

26 апреля 2023 г., 12:02:18 


\section{ПРИНЦИП ИНВАРИАНТНОСТИ ДЛЯ КРИТИЧЕСКОГО ВЕТВЯЩЕГОСЯ ПРОЦЕССА В СЛУЧАЙНОЙ СРЕДЕ, ДОСТИГАЮЩЕГО ВЫСОКОГО УРОВНЯ ${ }^{1)}$}

\footnotetext{
Установлен условный принцип инвариантности для критического ветвящегося процесса в случайной среде при условии достижения им высокого уровня и найдены конечномерные распределения предельного процесса.

Ключевые слова и фразы: ветвящийся процесс в случайной среде, условные принципы инвариантности, броуновская экскурсия, броуновская извилина.
}

1. Пусть $\left\{\xi_{n}, n \in \mathbf{N} \cup\{0\}\right\}$ - ветвящийся процесс в случайной среде (ВПСС), задаваемый последовательностью независимых одинаково распределенных (случайных) производящих функций $\left\{f_{n}(s), n \in \mathbf{N}\right\}$. Предполагается, что производящая функция $f_{n}(s), s \in[-1,1]$, определяет закон размножения частиц в $(n-1)$-м поколении, $n \in \mathbf{N}$, и $\xi_{0}=1$. Определение этого процесса можно найти, например, в [1].

Положим

$$
X_{n}=\ln f_{n}^{\prime}(1), \quad \eta_{n}=\frac{f_{n}^{\prime \prime}(1)}{\left(f_{n}^{\prime}(1)\right)^{2}}, \quad n \in \mathbf{N}
$$

Будем предполагать, что процесс $\left\{\xi_{n}\right\}$ является критическим, т.е.

$$
\mathbf{E} X_{1}=0
$$

и выполнены следующие моментные ограничения:

$$
0<\mathbf{E} X_{1}^{2}=: \sigma^{2}<+\infty, \quad \mathbf{E} \ln ^{q}\left(\eta_{1} \vee 1\right)<+\infty
$$

при некотором $q>2$.

\footnotetext{
* Математический институт им. В.А.Стеклова РАН, ул. Губкина, 8, 119991 Москва, ГСП-1, Россия; e-mail: viafan@mi.ras.ru

1) Работа выполнена при финансовой поддержке РФФИ (грант № 05-01-00035) и программы «Ведущие научные школы» (грант НШ-4129.2006.1).
} 
Рассмотрим момент вырождения процесса $\left\{\xi_{n}\right\}: T=\min \left\{n: \xi_{n}=0\right\}$. В [1], [2] установлено, что при выполнении условий (1), (2) и $n \rightarrow \infty$

$$
\mathbf{P}(T>n) \sim \frac{c_{1}}{\sqrt{n}}
$$

где $c_{1}$ - положительная постоянная, и

$$
\begin{gathered}
\left\{\frac{\ln \left(\xi_{\lfloor n t\rfloor} \vee 1\right)}{\sigma \sqrt{n}}, \frac{\ln \left(\xi_{\lfloor t T\rfloor} \vee 1\right)}{\sigma \sqrt{n}}, t \in[0,+\infty) \mid T>n\right\} \\
\stackrel{D}{\longrightarrow}\left\{W^{+}(t \wedge \tau), W^{+}(t \tau \wedge \tau), t \in[0,+\infty)\right\},
\end{gathered}
$$

где знак $\stackrel{D}{\longrightarrow}$ означает сходимость по распределению в функциональном пространстве $D[0,+\infty) \times D[0,+\infty)$ с борелевской $\sigma$-алгеброй относительно топологии Скорохода, случайный процесс $W^{+}=\left\{W^{+}(t), t \in\right.$ $[0,+\infty)\}$ является броуновской извилиной (причем при $t \geqslant 1$ процесс $W^{+}(t)-W^{+}(1)$ является стандартным броуновским движением, не зависящим от процесса $\left.\left\{W^{+}(t), t \in[0,1]\right\}\right)$ и $\tau=\inf \left\{t>1: W^{+}\{t)=0\right\}$.

Отметим, что

$$
\begin{aligned}
& \left\{W^{+}(t \wedge \tau), W^{+}(t \tau \wedge \tau), t \in[0,+\infty)\right\} \\
& \quad \stackrel{d}{=}\left\{\frac{W_{0}^{+}\left(\alpha^{2} t\right)}{\alpha}, \frac{W_{0}^{+}(t)}{\alpha}, t \in[0,+\infty)\right\},
\end{aligned}
$$

где случайный процесс $W_{0}^{+}=\left\{W_{0}^{+}(t), t \in[0,+\infty)\right\}$ является броуновской экскурсией, причем $W_{0}^{+}(t)=0$ при $t \geqslant 1$ и $\alpha$ - случайная величина, равномерно распределенная на интервале $(0,1)$ и не зависящая от процесса $W_{0}^{+}$.

Рассмотрим теперь момент первого достижения уровня $x>0$ процессом $\left\{\xi_{n}\right\}$ :

$$
T_{x}=\min \left\{n: \xi_{n}>x\right\} .
$$

В указанном принципе инвариантности (4) рассматривается ВПСС, удовлетворяющий условию $\{T>n\}, n \rightarrow \infty$, т.е. долгоживущий ВПСС. Наша задача - получить принцип инвариантности для критического ВПСС, достигающего высокого уровня, т.е. удовлетворяющего условию $\left\{T_{x}<+\infty\right\}, x \rightarrow+\infty$.

Заметим, что в работах автора [2], [3] установлено, что если выполнены условия (1), (2), то при $x \rightarrow+\infty$

$$
\mathbf{P}\left(T_{x}<+\infty\right) \sim \frac{c_{2}}{\ln x},
$$

где $c_{2}=c_{1} \sigma \sqrt{\pi / 2}$, и при тех же условиях доказаны предельные теоремы для случайных величин $T_{x} / \ln ^{2} x, T_{x} / T, T / \ln ^{2} x, \mu(x) / \ln ^{2} x, \nu(x) / \ln ^{2} x$, $\mu(x) / T, \nu(x) / T$ (здесь $\mu(x)$ и $\nu(x)$ - мощности множеств $\left\{i: \xi_{i}>x\right\}$ и 
$\left\{i: 0<\xi_{i} \leqslant x\right\}$ соответственно), рассматриваемых при условии $T(x)<$ $+\infty$.

Сформулируем основные результаты. Положим $h^{+}=\sup _{t \in[0,+\infty)} W^{+}(t)$, $h_{0}^{+}=\sup _{t \in[0,1]} W_{0}^{+}(t)$ и для каждого $v \in(0,+\infty)$ введем случайный процесс $Z_{v}$ :

$$
Z_{v}(t)=\frac{W_{0}^{+}\left(v^{2} t\right)}{v}, \quad t \in[0,+\infty) .
$$

Теорема 1. При выполнении условий (1), (2) и $x \rightarrow+\infty$

$$
\left\{\frac{\ln \left(\xi_{\left\lfloor t \sigma^{-2} x^{2}\right\rfloor} \vee 1\right)}{x}, t \in[0,+\infty) \mid T_{\exp (x)}<+\infty\right\} \stackrel{D}{\longrightarrow} Y,
$$

где $Y=\{Y(t), t \in[0,+\infty)\}-$ случайньй прочесс с непрерывнылми траекториями, распределение которого задается формулой

$$
\mathbf{P}(Y \in A)=\sqrt{\frac{2}{\pi}} \int_{0}^{+\infty} \mathbf{P}\left(Z_{v} \in A, h_{0}^{+}>v\right) d v
$$

для любого борелевского множества А пространства $D[0,+\infty)$.

В этой теореме знак $\stackrel{D}{\longrightarrow}$ означает сходимость по распределению в функциональном пространстве $D[0,+\infty)$ с борелевской $\sigma$-алгеброй относительно топологии Скорохода.

Очевидно, что процесс $Y$ стартует из состояния 0, его траектории п.н. неотрицательны, причем при новом попадании в состояние 0 процесс остается там навсегда.

Прежде чем сформулировать следующую теорему, в которой рассматриваются конечномерные распределения предельного процесса $Y$, введем некоторые обозначения:

1) при $t>0, x>0, y>0$

$$
\begin{aligned}
f(t, x) & =\sqrt{\frac{2}{\pi t^{3}}} x \exp \left(-\frac{x^{2}}{2 t}\right) \\
g(t, x, y) & =\frac{1}{\sqrt{2 \pi t}}\left[\exp \left(-\frac{(y-x)^{2}}{2 t}\right)-\exp \left(-\frac{(y+x)^{2}}{2 t}\right)\right]
\end{aligned}
$$

2) при $t>0, y \in(0,1]$

$$
p_{1}(0,0 ; t, y)=\sum_{k=-\infty}^{+\infty} f(t, 2 k+y)
$$

при $0<s<t, x \in(0,1], y \in(0,1]$

$$
p_{1}(s, x ; t, y)=\sum_{k=-\infty}^{+\infty} g(t-s, x, 2 k+y) ;
$$


3) при $t>0$

$$
p_{2}(0,0 ; t, y)= \begin{cases}\sum_{k \in \mathbf{Z} \backslash\{0\}} f(t, 2 k-y), & y \in(0,1], \\ f(t, y), & y>1,\end{cases}
$$

при $0<s<t, x \in(0,1]$

$$
p_{2}(s, x ; t, y)= \begin{cases}\sum_{k \in \mathbf{Z} \backslash\{0\}} g(t-s,-x, 2 k+y), & y \in(0,1], \\ g(t-s, x, y), & y>1 .\end{cases}
$$

Теорема 2. Для произвольных чисел $m \in \mathbf{N}, t_{1}, t_{2}, \ldots, t_{m} \in(0,+\infty)$ $\left(t_{1}<t_{2}<\cdots<t_{m}\right) u a_{1}, a_{2}, \ldots, a_{m} \in[0,1)$

$$
\begin{aligned}
\mathbf{P} & \left.Y\left(t_{i}\right)>a_{i}, i=1, \ldots, m\right) \\
= & \sum_{j=0}^{m} \int_{G_{j}\left(a_{1}, \ldots, a_{m}\right)} \ldots \prod_{i=0}^{j-1} p_{1}\left(t_{i}, x_{i} ; t_{i+1}, x_{i+1}\right) p_{2}\left(t_{j}, x_{j} ; t_{j+1}, x_{j+1}\right) \\
& \times \prod_{i=j+1}^{m-1} g\left(t_{i+1}-t_{i}, x_{i}, x_{i+1}\right) d x_{1} \cdots d x_{m},
\end{aligned}
$$

где $t_{0}=0, x_{0}=0, t_{m+1}=+\infty, x_{m+1}=+\infty, p_{2}\left(t_{m}, x_{m} ; t_{m+1}, x_{m+1}\right)=x_{m} u$

$$
\begin{aligned}
& G_{j}\left(a_{1}, \ldots, a_{m}\right)=\left\{\left(x_{1}, \ldots, x_{m}\right): a_{i}<x_{i} \leqslant 1, i=1, \ldots, j ; x_{i}>a_{i},\right. \\
&i=j+1, \ldots, m\} \quad \text { npu } j=0,1, \ldots, m-1, \\
& G_{0}\left(a_{1}, \ldots, a_{m}\right)=\left\{\left(x_{1}, \ldots, x_{m}\right): x_{i}>a_{i}, i=1, \ldots, m\right\}, \\
& G_{m}\left(a_{1}, \ldots, a_{m}\right)=\left\{\left(x_{1}, \ldots, x_{m}\right): a_{i}<x_{i} \leqslant 1, i=1, \ldots, m\right\} .
\end{aligned}
$$

Заметим, что, как обычно, произведение по пустому множеству индексов равно единице. В представленной формуле для конечномерного распределения процесса $Y$ имеется $m+1$ слагаемое, каждое из них устроено аналогично конечномерному распределению марковского процесса, при этом в качестве переходных плотностей фигурируют $p_{1}(s, x ; t, y)$, $p_{2}(s, x ; t, y)$ и $g(t, x, y)$.

2. Доказательство теоремы 1. Для каждого $x \in(1,+\infty)$ введем случайный процесс $Y_{x}$ :

$$
Y_{x}(t)=\frac{\ln \left(\xi_{\left\lfloor t \sigma^{-2} \ln ^{2} x\right\rfloor} \vee 1\right)}{\ln x}, \quad t \in[0,+\infty) .
$$

Требуется показать, что для произвольного борелевского множества $A$ из $D[0,+\infty)$, удовлетворяющего условию $\mathbf{P}(Y \in \partial A)=0(\partial A-$ граница множества $A$ ), справедливо равенство

$$
\lim _{x \rightarrow+\infty} \mathbf{P}\left(Y_{x} \in A \mid T_{x}<+\infty\right)=\mathbf{P}(Y \in A) .
$$


Заметим, что для произвольного $\varepsilon>0$

$$
\mathbf{P}\left(Y_{x} \in A, T_{x}<+\infty\right)=P_{1}(x, \varepsilon)+P_{2}(x, \varepsilon),
$$

где

$$
\begin{aligned}
& P_{1}(x, \varepsilon)=\mathbf{P}\left(Y_{x} \in A, T_{x}<+\infty, T>\varepsilon \sigma^{-2} \ln ^{2} x\right), \\
& P_{2}(x, \varepsilon)=\mathbf{P}\left(Y_{x} \in A, T_{x}<+\infty, T \leqslant \varepsilon \sigma^{-2} \ln ^{2} x\right) .
\end{aligned}
$$

Существенную роль в дальнейшем играет следующее утверждение, доказанное в работе [2].

Лемма 1. При выполнении условий (1), (2)

$$
\lim _{\varepsilon \downarrow 0} \limsup _{x \rightarrow+\infty} \mathbf{P}\left(T \leqslant \varepsilon \ln ^{2} x \mid T_{x}<+\infty\right)=0 .
$$

Поскольку $P_{2}(x, \varepsilon) \leqslant \mathbf{P}\left(T_{x}<+\infty, T \leqslant \varepsilon \sigma^{-2} \ln ^{2} x\right)$, то в силу леммы 1

$$
\lim _{\varepsilon \downarrow 0} \limsup _{x \rightarrow+\infty} \frac{P_{2}(x, \varepsilon)}{\mathbf{P}\left(T_{x}<+\infty\right)}=0 .
$$

Поскольку

$$
\left\{T_{x}<+\infty\right\}=\left\{\sup _{t \in[0,1]} \xi_{\lfloor t T\rfloor}>x\right\}=\left\{\sup _{t \in[0,1]} \ln \left(\xi_{\lfloor t T\rfloor} \vee 1\right)>\ln x\right\}
$$

то, полагая $n=\varepsilon \sigma^{-2} \ln ^{2} x$ (точнее было бы написать $\left.\left\lfloor\varepsilon \sigma^{-2} \ln ^{2} x\right\rfloor\right)$, получаем, что

$$
\begin{array}{r}
P_{1}(x, \varepsilon)=\mathbf{P}\left(\left\{\sqrt{\varepsilon} \frac{\ln \left(\xi_{\lfloor n t / \varepsilon\rfloor} \vee 1\right)}{\sigma \sqrt{n}}, t \geqslant 0\right\} \in A,\right. \\
\left.\sup _{t \in[0,1]}\left(\xi_{\lfloor t T\rfloor} \vee 1\right)>\frac{\sigma \sqrt{n}}{\sqrt{\varepsilon}}, T>n\right) .
\end{array}
$$

Заметим, что при $n=\varepsilon \sigma^{-2} \ln ^{2} x$, ввиду соотношений (3) и (6),

$$
\lim _{x \rightarrow+\infty} \frac{\mathbf{P}(T>n)}{\mathbf{P}\left(T_{x}<+\infty\right)}=\frac{\sigma c_{1}}{\sqrt{\varepsilon} c_{2}}=\sqrt{\frac{2}{\varepsilon \pi}} .
$$

Применяя к правой части (11) принцип инвариантности (4) и используя соотношения (5) и (12), находим, что

$$
\begin{aligned}
\lim _{x \rightarrow+\infty} & \frac{P_{1}(x, \varepsilon)}{\mathbf{P}\left(T_{x}<+\infty\right)} \\
= & \sqrt{\frac{2}{\varepsilon \pi}} \mathbf{P}\left(\left\{\sqrt{\varepsilon} \frac{W_{0}^{+}\left(\alpha^{2} t / \varepsilon\right)}{\alpha}, t \geqslant 0\right\} \in A, \sup _{t \in[0,1]} \frac{W_{0}^{+}(t)}{\alpha}>\frac{1}{\sqrt{\varepsilon}}\right) .
\end{aligned}
$$


Преобразуем вероятность в правой части (13), учитывая, что случайная величина $\alpha$ равномерно распределена на интервале $(0,1)$ и не зависит от процесса $W_{0}^{+}$:

$$
\begin{aligned}
\mathbf{P}(\{ & \left.\left.\sqrt{\varepsilon} \frac{W_{0}^{+}\left(\alpha^{2} t / \varepsilon\right)}{\alpha}, t \geqslant 0\right\} \in A, \sup _{t \in[0,1]} \frac{W_{0}^{+}(t)}{\alpha}>\frac{1}{\sqrt{\varepsilon}}\right) \\
& =\int_{0}^{1} \mathbf{P}\left(\left\{\sqrt{\varepsilon} \frac{W_{0}^{+}\left(u^{2} t / \varepsilon\right)}{u}, t \geqslant 0\right\} \in A, \sup _{t \in[0,1]} \frac{W_{0}^{+}(t)}{u}>\frac{1}{\sqrt{\varepsilon}}\right) d u \\
& =\sqrt{\varepsilon} \int_{0}^{1 / \sqrt{\varepsilon}} \mathbf{P}\left(\left\{\frac{W_{0}^{+}\left(v^{2} t\right)}{v}, t \geqslant 0\right\} \in A, \sup _{t \in[0,1]} W_{0}^{+}(t)>v\right) d v .
\end{aligned}
$$

В итоге, вспоминая определение процесса $Z_{v}$ и случайной величины $h_{0}^{+}$, получаем, что

$$
\lim _{x \rightarrow+\infty} \frac{P_{1}(x, \varepsilon)}{\mathbf{P}\left(T_{x}<+\infty\right)}=\sqrt{\frac{2}{\pi}} \int_{0}^{1 / \sqrt{\varepsilon}} \mathbf{P}\left(Z_{v} \in A, h_{0}^{+}>v\right) d v .
$$

Переходя теперь к пределу при $\varepsilon \downarrow 0$, видим, что

$$
\lim _{\varepsilon \downarrow 0} \lim _{x \rightarrow+\infty} \frac{P_{1}(x, \varepsilon)}{\mathbf{P}\left(T_{x}<+\infty\right)}=\sqrt{\frac{2}{\pi}} \int_{0}^{+\infty} \mathbf{P}\left(Z_{v} \in A, h_{0}^{+}>v\right) d v .
$$

Из соотношений (8), (9) и (14) вытекает равенство

$$
\lim _{x \rightarrow+\infty} \mathbf{P}\left(Y_{x} \in A \mid T_{x}<+\infty\right)=\sqrt{\frac{2}{\pi}} \int_{0}^{+\infty} \mathbf{P}\left(Z_{v} \in A, h_{0}^{+}>v\right) d v
$$

что и означает справедливость соотношения (7).

Однако в приведенных рассуждениях есть изъян, поскольку ключевое соотношение (13) справедливо, если $\mathbf{P}\left(\left\{\sqrt{\varepsilon} W_{0}^{+}\left(\alpha^{2} t \varepsilon^{-1}\right) \alpha^{-1}, t \geqslant 0\right\} \in\right.$ $\partial A)=0$; нам же известно, что $\mathbf{P}(Y \in \partial A)=0$.

Чтобы обойти эту трудность, рассмотрим сначала цилиндрические подмножества $D[0,+\infty): A=\left\{y: y\left(t_{i}\right) \leqslant a_{i}, i=1, \ldots, m\right\}$, где $m \in \mathbf{N}$, $t_{1}, t_{2}, \ldots, t_{m} \in[0,+\infty)$ и $a_{1}, a_{2}, \ldots, a_{m} \in \mathbf{R}$. Для них соотношения (13) и (следовательно) (15) справедливы ввиду абсолютной непрерывности конечномерных распределений броуновской экскурсии.

Теперь рассмотрим подмножества $D[0,+\infty)$ вида

$$
A=\left\{y: w_{y}(\delta ; a, b) \geqslant \varepsilon\right\},
$$

где $0 \leqslant a<b<+\infty, \varepsilon, \delta-$ положительные числа, $w_{y}(\delta ; a, b)-$ стандартный модуль непрерывности для функции $y(t), t \in[0,+\infty)$, т.е.

$$
w_{y}(\delta ; a, b)=\sup _{t, s:|t-s| \leqslant \delta}|y(t)-y(s)| \quad(t, s \in[a, b]) .
$$


Нетрудно понять, что отображение $y \mapsto w_{y}(\delta ; a, b), y \in D[0,+\infty)$, непрерывно, если $y(t)-$ непрерывная функция на $[0,+\infty)$. Учитывая, что почти все траектории броуновской экскурсии непрерывны, убеждаемся в справедливости соотношения (13) для рассматриваемых множеств $A$ и всех положительных $\varepsilon$, за исключением не более чем счетного множества. Но это означает, что выполнено соотношение (15), которое можно переписать в виде

$$
\begin{aligned}
& \lim _{x \rightarrow+\infty} \mathbf{P}\left(w_{Y_{x}}(\delta ; a, b) \geqslant \varepsilon \mid T_{x}<+\infty\right) \\
& \quad=\sqrt{\frac{2}{\pi}} \int_{0}^{+\infty} \mathbf{P}\left(w_{Z_{v}}(\delta ; a, b) \geqslant \varepsilon, h_{0}^{+}>v\right) d v .
\end{aligned}
$$

Известно (см. [4]), что

$$
\int_{0}^{+\infty} \mathbf{P}\left(h_{0}^{+}>v\right) d v=\mathbf{E} h_{0}^{+}=\sqrt{\frac{\pi}{2}} .
$$

Далее, ввиду непрерывности почти всех траекторий процесса $Z_{v}$

$$
\lim _{\delta \rightarrow 0} \mathbf{P}\left(w_{Z_{v}}(\delta ; a, b) \geqslant \varepsilon\right)=0 .
$$

Следовательно, по теореме о мажорируемой сходимости из соотношения (16) получаем, что

$$
\lim _{\delta \rightarrow 0} \lim _{x \rightarrow+\infty} \mathbf{P}\left(w_{Y_{x}}(\delta ; a, b) \geqslant \varepsilon \mid T_{x}<+\infty\right)=0 .
$$

Но сходимость конечномерных распределений процесса $Y_{x}$ и соотношение (17) в силу теоремы 15.5 из [5] означают справедливость теоремы 1 (включая утверждение о непрерывности траекторий процесса $Y$ ).

3. Доказательство теоремы 2. Поскольку по условию теоремы все числа $a_{1}, \ldots, a_{m}$ неотрицательны, то, учитывая соотношение (10), получаем, что

$$
\begin{aligned}
\mathbf{P} & \left(Y_{x}\left(t_{i}\right)>a_{i}, i=1, \ldots, m \mid T_{x}<+\infty\right) \\
= & \mathbf{P}\left(Y_{x}\left(t_{i}\right)>a_{i}, i=1, \ldots, m ; T>\frac{t_{m}}{\sigma^{2}} \ln ^{2} x \mid T_{x}<+\infty\right) \\
= & \mathbf{P}\left(Y_{x}\left(t_{i}\right)>a_{i}, i=1, \ldots, m ; \sup _{t \in[0,1]} \ln \left(\xi_{\lfloor t T\rfloor} \vee 1\right)>\ln x \mid T>\frac{t_{m}}{\sigma^{2}} \ln ^{2} x\right) \\
& \times \frac{\mathbf{P}\left(T>t_{m} \sigma^{-2} \ln ^{2} x\right)}{\mathbf{P}\left(T_{x}<+\infty\right)} .
\end{aligned}
$$

Откуда, полагая $n=t_{m} \sigma^{-2} \ln ^{2} x$ и используя принцип инвариантности (4) и соотношение (12), находим, что при любых неотрицательных $a_{1}, \ldots, a_{m}$

$$
\begin{aligned}
& \lim _{x \rightarrow+\infty} \mathbf{P}\left(Y_{x}\left(t_{i}\right)>a_{i}, i=1, \ldots, m \mid T_{x}<+\infty\right) \\
& \quad=\sqrt{\frac{2}{\pi t_{m}}} \mathbf{P}\left(W^{+}\left(\frac{t_{i}}{t_{m}}\right)>\frac{a_{i}}{\sqrt{t_{m}}}, i=1, \ldots, m ; h^{+}>\frac{1}{\sqrt{t_{m}}}\right)
\end{aligned}
$$


(здесь также учтено, что совместное распределение случайных величин, стоящих под знаком последней вероятности, является абсолютно непрерывным). Но это, в силу теоремы 1 , означает, что при всех неотрицательных $a_{1}, \ldots, a_{m}$

$$
\begin{aligned}
& \mathbf{P}\left(Y\left(t_{i}\right)>a_{i}, i=1, \ldots, m\right) \\
& \quad=\sqrt{\frac{2}{\pi t_{m}}} \mathbf{P}\left(W^{+}\left(\frac{t_{i}}{t_{m}}\right)>\frac{a_{i}}{\sqrt{t_{m}}}, i=1, \ldots, m ; h^{+}>\frac{1}{\sqrt{t_{m}}}\right) .
\end{aligned}
$$

Введем момент первого достижения уровня $x>0$ для процесса $\left\{W^{+}(t \wedge \tau), t \geqslant 0\right\}$ (имеющего непрерывные траектории):

$$
\tau_{x}^{+}=\inf \left\{t: W^{+}(t \wedge \tau)=x\right\} .
$$

Запишем следующее представление для вероятности в правой части (18):

$$
\mathbf{P}\left(W^{+}\left(\frac{t_{i}}{t_{m}}\right)>\frac{a_{i}}{\sqrt{t_{m}}}, i=1, \ldots, m ; h^{+}>\frac{1}{\sqrt{t_{m}}}\right)=\sum_{j=0}^{m} P_{j},
$$

где

$$
P_{j}=\mathbf{P}\left(W^{+}\left(\frac{t_{i}}{t_{m}}\right)>\frac{a_{i}}{\sqrt{t_{m}}}, i=1, \ldots, m ; \tau_{1 / \sqrt{t_{m}}}^{+} \in\left[\frac{t_{j}}{t_{m}}, \frac{t_{j+1}}{t_{m}}\right)\right) .
$$

Положим при $t>0, a<b$

$$
N_{t}(a, b)=\frac{1}{\sqrt{2 \pi t}} \int_{a}^{b} \exp \left(\frac{-u^{2}}{2 t}\right) d u .
$$

Известно (см., например, [4]), что броуновская извилина является неоднородным марковским процессом с переходной плотностью $p^{+}(s, x ; t, y)$ : при $t \in(0,1], y>0$

$$
p^{+}(0,0 ; t, y)=\sqrt{2 \pi} f(t, y) N_{1-t}(0, y),
$$

а при $0<s<t \leqslant 1, x>0, y>0$

$$
p^{+}(s, x ; t, y)=g(t-s, x, y) \frac{N_{1-t}(0, y)}{N_{1-s}(0, x)} .
$$

Поэтому при $j=0,1, \ldots, m-1$

$$
\begin{aligned}
P_{j}= & \int_{G_{j}\left(a_{1}, \ldots, a_{m}\right)} \ldots \prod_{i=0}^{j-1} \mathbf{P}^{\left(t_{i}, x_{i}\right)}\left(W^{+}\left(\frac{t_{i+1}}{t_{m}}\right) \in \frac{d x_{i+1}}{\sqrt{t_{m}}}, \sup _{t \in\left[t_{i}, t_{i+1}\right]} W^{+}\left(\frac{t}{t_{m}}\right) \leqslant \frac{1}{\sqrt{t_{m}}}\right) \\
& \times \mathbf{P}^{\left(t_{j}, x_{j}\right)}\left(W^{+}\left(\frac{t_{j+1}}{t_{m}}\right) \in \frac{d x_{j+1}}{\sqrt{t_{m}}}, \sup _{t \in\left[t_{j}, t_{j+1}\right]} W^{+}\left(\frac{t}{t_{m}}\right)>\frac{1}{\sqrt{t_{m}}}\right) \\
& \times \prod_{i=j+1}^{m-1} \mathbf{P}^{\left(t_{i}, x_{i}\right)}\left(W^{+}\left(\frac{t_{i+1}}{t_{m}}\right) \in \frac{d x_{i+1}}{\sqrt{t_{m}}}\right)
\end{aligned}
$$


где символ $\mathbf{P}^{\left(t_{i}, x_{i}\right)}(A)$ означает условную вероятность события $A$ при условии, что $W^{+}\left(t_{i} / t_{m}\right)=x_{i} / \sqrt{t_{m}}$.

Очевидно, что

$$
\mathbf{P}^{\left(t_{i}, x_{i}\right)}\left(W^{+}\left(\frac{t_{i+1}}{t_{m}}\right) \in \frac{d x_{i+1}}{\sqrt{t_{m}}}\right)=p^{+}\left(\frac{t_{i}}{t_{m}}, \frac{x_{i}}{\sqrt{t_{m}}} ; \frac{t_{i+1}}{t_{m}}, \frac{x_{i+1}}{\sqrt{t_{m}}}\right) \frac{d x_{i+1}}{\sqrt{t_{m}}} .
$$

Далее, в работе [4] показано, что при $t \in(0,1], u>0,0<v \leqslant u$

$$
\mathbf{P}\left(W^{+}(t) \in d v, \sup _{s \in[0, t]} W^{+}(s) \leqslant u\right)=\sqrt{2 \pi} N_{1-t}(0, v) \sum_{k=-\infty}^{+\infty} f(t, 2 k u+v) d v
$$

поэтому при $0<x_{1} \leqslant 1$

$$
\begin{aligned}
\mathbf{P}^{\left(t_{0}, x_{0}\right)} & \left(W^{+}\left(\frac{t_{1}}{t_{m}}\right) \in \frac{d x_{1}}{\sqrt{t_{m}}}, \sup _{t \in\left[t_{0}, t_{1}\right]} W^{+}\left(\frac{t}{t_{m}}\right) \leqslant \frac{1}{\sqrt{t_{m}}}\right) \\
= & \sqrt{2 \pi} N_{1-t_{1} / t_{m}}\left(0, \frac{x_{1}}{\sqrt{t_{m}}}\right) \sum_{k=-\infty}^{+\infty} f\left(\frac{t_{1}}{t_{m}}, \frac{2 k+x_{1}}{\sqrt{t_{m}}}\right) \frac{d x_{1}}{\sqrt{t_{m}}} \\
= & \sqrt{2 \pi t_{m}} N_{1-t_{1} / t_{m}}\left(0, \frac{x_{1}}{\sqrt{t_{m}}}\right) \sum_{k=-\infty}^{+\infty} f\left(t_{1}, 2 k+x_{1}\right) d x_{1},
\end{aligned}
$$

причем последнее равенство объясняется тем, что

$$
f\left(\frac{t_{1}}{t_{m}}, \frac{2 k+x_{1}}{\sqrt{t_{m}}}\right)=t_{m} f\left(t_{1}, 2 k+x_{1}\right) .
$$

Очевидно, что

$$
\begin{aligned}
\mathbf{P}^{\left(t_{0}, x_{0}\right)} & \left(W^{+}\left(\frac{t_{1}}{t_{m}}\right) \in \frac{d x_{1}}{\sqrt{t_{m}}}, \sup _{t \in\left[t_{0}, t_{1}\right]} W^{+}\left(\frac{t}{t_{m}}\right)>\frac{1}{\sqrt{t_{m}}}\right) \\
= & \mathbf{P}^{\left(t_{0}, x_{0}\right)}\left(W^{+}\left(\frac{t_{1}}{t_{m}}\right) \in \frac{d x_{1}}{\sqrt{t_{m}}}\right) \\
& -\mathbf{P}^{\left(t_{0}, x_{0}\right)}\left(W^{+}\left(\frac{t_{1}}{t_{m}}\right) \in \frac{d x_{1}}{\sqrt{t_{m}}}, \sup _{t \in\left[t_{0}, t_{1}\right]} W^{+}\left(\frac{t}{t_{m}}\right) \leqslant \frac{1}{\sqrt{t_{m}}}\right),
\end{aligned}
$$

поэтому с учетом $(20),(23)$ и $(25)$

$$
\begin{aligned}
\mathbf{P}^{\left(t_{0}, x_{0}\right)} & \left(W^{+}\left(\frac{t_{1}}{t_{m}}\right) \in \frac{d x_{1}}{\sqrt{t_{m}}}, \sup _{t \in\left[t_{0}, t_{1}\right]} W^{+}\left(\frac{t}{t_{m}}\right)>\frac{1}{\sqrt{t_{m}}}\right) \\
= & \sqrt{2 \pi t_{m}} N_{1-t_{1} / t_{m}}\left(0, \frac{x_{1}}{\sqrt{t_{m}}}\right) d x_{1} \\
& \times \begin{cases}\sum_{k \in \mathbf{Z} \backslash\{0\}} f\left(t_{1}, 2 k-x_{1}\right), & x_{1} \in(0,1], \\
f\left(t_{1}, x_{1}\right), & x_{1}>1 .\end{cases}
\end{aligned}
$$


Лемма 2. Для любых $t_{1}, t_{2} \in(0,1]\left(t_{1}<t_{2}\right)$, положительных $x_{1}, x_{2}$ и любого борелевского множества $A$ из пространства $D\left[t_{1}, t_{2}\right]$ справедливо равенство

$$
\begin{aligned}
& \mathbf{P}\left(W^{+} \in A \mid W^{+}\left(t_{1}\right)=x_{1}, W^{+}\left(t_{2}\right)=x_{2}\right) \\
& \quad=\mathbf{P}\left(W \in A \mid W\left(t_{1}\right)=x_{1}, W\left(t_{2}\right)=x_{2}, \inf _{t \in\left[t_{1}, t_{2}\right]} W(t) \geqslant 0\right),
\end{aligned}
$$

где $W=\{W(t), t \in[0,+\infty)\}-$ стандартное броуновское движение.

Д о к а з а т е л ь с т в о. Ограничимся рассмотрением таких множеств $A$, что $\mathbf{P}\left(W^{+} \in \partial A\right)=\mathbf{P}(W \in \partial A)=0$. Рассмотрим для произвольных положительных $a_{1}, a_{2}$ вероятность

$$
P^{*}=\mathbf{P}\left(W^{+}\left(t_{1}\right)>a_{1}, W^{+}\left(t_{2}\right)>a_{2}, W^{+} \in A\right) .
$$

Поскольку $W^{+}$является марковским процессом, то

$$
\begin{aligned}
P^{*}= & \int_{a 1}^{+\infty} \mathbf{P}\left(W^{+}\left(t_{1}\right) \in d x_{1}\right) \\
& \times \int_{a_{2}}^{+\infty} \mathbf{P}\left(W^{+}\left(t_{2}\right) \in d x_{2}, W^{+} \in A \mid W^{+}\left(t_{1}\right)=x_{1}\right) .
\end{aligned}
$$

Напомним принцип инвариантности Иглхарта (см. [6], [7]): для случайного блуждания $\left\{S_{n}, n \in \mathbf{N}\right\}$ с нулевым сносом и конечной шаговой дисперсией $\sigma^{2}>0$ при $n \rightarrow \infty$

$$
\left\{\frac{S_{\lfloor n t\rfloor}}{\sigma \sqrt{n}}, t \in[0,+\infty) \mid \min _{t \in[0,1]} S_{\lfloor n t\rfloor} \geqslant 0\right\} \stackrel{D}{\longrightarrow} W^{+} .
$$

В силу этого результата

$$
P^{*}=\lim _{n \rightarrow \infty} \mathbf{P}\left(\frac{S_{\left\lfloor n t_{i}\right\rfloor}}{\sigma \sqrt{n}}>a_{i}, i=1,2 ;\left\{\frac{S_{\lfloor n t\rfloor}}{\sigma \sqrt{n}}, t \geqslant 0\right\} \in A \mid \min _{t \in[0,1]} S_{\lfloor n t\rfloor} \geqslant 0\right) .
$$

Заметим, что

$$
\begin{aligned}
& \mathbf{P}\left(\frac{S_{\left\lfloor n t_{i}\right\rfloor}}{\sigma \sqrt{n}}>a_{i}, i=1,2 ;\left\{\frac{S_{\lfloor n t\rfloor}}{\sigma \sqrt{n}}, t \geqslant 0\right\} \in A \mid \min _{t \in[0,1]} S_{\lfloor n t\rfloor} \geqslant 0\right) \\
& =\frac{\mathbf{P}\left(\min _{t \in\left[0, t_{1}\right]} S_{\lfloor n t\rfloor} \geqslant 0\right)}{\mathbf{P}\left(\min _{t \in[0,1]} S_{\lfloor n t\rfloor} \geqslant 0\right)} \int_{a_{1}}^{+\infty} \mathbf{P}\left(\frac{S_{\left\lfloor n t_{1}\right\rfloor}}{\sigma \sqrt{n}} \in d x_{1} \mid \min _{t \in\left[0, t_{1}\right]} S_{\lfloor n t\rfloor} \geqslant 0\right) \\
& \quad \times \int_{a_{2}}^{+\infty} \mathbf{P}\left(\frac{S_{\left\lfloor n t_{2}\right\rfloor}}{\sigma \sqrt{n}} \in d x_{2},\left\{\frac{S_{\lfloor n t\rfloor}}{\sigma \sqrt{n}}, t \geqslant 0\right\} \in A,\right. \\
& \left.\quad \times \mathbf{P} \min _{t \in\left[t_{1}, t_{2}\right\rfloor} S_{\lfloor n t\rfloor} \geqslant 0 \mid \frac{S_{\left\lfloor n t_{1}\right\rfloor}}{\sigma \sqrt{n}}=x_{1}\right) \\
& \left.\quad \times \min _{t \in\left[t_{2}, 1\right]} S_{\lfloor n t\rfloor} \geqslant 0 \mid \frac{S_{\left\lfloor n t_{2}\right\rfloor}}{\sigma \sqrt{n}}=x_{2}\right) .
\end{aligned}
$$


Первая дробь в правой части равенства (29) стремится при $n \rightarrow \infty$ к $1 / \sqrt{t_{1}}$, поскольку (см. [9, гл. 12]) при $n \rightarrow \infty$

$$
\mathbf{P}\left(\min _{t \in[0,1]} S_{\lfloor n t\rfloor} \geqslant 0\right) \sim \frac{c_{3}}{\sqrt{n}},
$$

где $c_{3}$ - положительная постоянная. Теперь рассмотрим три вероятности под знаком интеграла в правой части соотношения (29). Первая вероятность по принципу инвариантности Иглхарта стремится при $n \rightarrow \infty$ к $\mathbf{P}\left(W^{+}(1) \in d x_{1} / \sqrt{t_{1}}\right)$. Вторая же вероятность по принципу инвариантности Прохорова-Донскера стремится при $n \rightarrow \infty \mathrm{K}$

$$
\mathbf{P}\left(W\left(t_{2}\right) \in d x_{2}, W \in A, \inf _{t \in\left[t_{1}, t_{2}\right]} W(t) \geqslant 0 \mid W\left(t_{1}\right)=x_{1}\right) .
$$

Наконец, третья вероятность по тому же принципу инвариантности стремится при $n \rightarrow \infty \mathrm{K} \mathbf{P}\left(\inf _{t \in\left[t_{2}, 1\right]} W(t) \geqslant 0 \mid W\left(t_{2}\right)=x_{2}\right)$. Из соотношений $(28),(29)$ и последних утверждений следует, что

$$
\begin{aligned}
P^{*}= & \int_{a_{1}}^{+\infty} \frac{1}{\sqrt{t_{1}}} \mathbf{P}\left(W^{+}(1) \in \frac{d x_{1}}{\sqrt{t_{1}}}\right) \int_{a_{2}}^{+\infty} \mathbf{P}\left(\inf _{t \in\left[t_{2}, 1\right]} W(t) \geqslant 0 \mid W\left(t_{2}\right)=x_{2}\right) \\
& \times \mathbf{P}\left(W\left(t_{2}\right) \in d x_{2}, W \in A, \inf _{t \in\left[t_{1}, t_{2}\right]} W(t) \geqslant 0 \mid W\left(t_{1}\right)=x_{1}\right) .
\end{aligned}
$$

Сравнивая формулы (27) и (30), видим, что

$$
\begin{aligned}
\mathbf{P}\left(W^{+}\left(t_{1}\right) \in d x_{1}\right) \mathbf{P}\left(W^{+}\left(t_{2}\right) \in d x_{2}, W^{+} \in A \mid W^{+}\left(t_{1}\right)=x_{1}\right) \\
=\frac{1}{\sqrt{t_{1}}} \mathbf{P}\left(W^{+}(1) \in \frac{d x_{1}}{\sqrt{t_{1}}}\right) \mathbf{P}\left(\inf _{t \in\left[t_{2}, 1\right]} W(t) \geqslant 0 \mid W\left(t_{2}\right)=x_{2}\right) \\
\quad \times \mathbf{P}\left(W\left(t_{2}\right) \in d x_{2}, W \in A, \inf _{t \in\left[t_{1}, t_{2}\right]} W(t) \geqslant 0 \mid W\left(t_{1}\right)=x_{1}\right) .
\end{aligned}
$$

Выбирая в качестве $A$ достоверное событие, получаем, что

$$
\begin{aligned}
\mathbf{P} & \left(W^{+}\left(t_{1}\right) \in d x_{1}\right) \mathbf{P}\left(W^{+}\left(t_{2}\right) \in d x_{2} \mid W^{+}\left(t_{1}\right)=x_{1}\right) \\
= & \frac{1}{\sqrt{t_{1}}} \mathbf{P}\left(W^{+}(1) \in \frac{d x_{1}}{\sqrt{t_{1}}}\right) \mathbf{P}\left(\inf _{t \in\left[t_{2}, 1\right]} W(t) \geqslant 0 \mid W\left(t_{2}\right)=x_{2}\right) \\
& \times \mathbf{P}\left(W\left(t_{2}\right) \in d x_{2}, \inf _{t \in\left[t_{1}, t_{2}\right]} W(t) \geqslant 0 \mid W\left(t_{1}\right)=x_{1}\right) .
\end{aligned}
$$

Приравняв частное от деления правых частей (31) и (32) к частному от деления левых частей, получаем, что

$$
\begin{aligned}
& \frac{\mathbf{P}\left(W^{+}\left(t_{2}\right) \in d x_{2}, W^{+} \in A \mid W^{+}\left(t_{1}\right)=x_{1}\right)}{\mathbf{P}\left(W^{+}\left(t_{2}\right) \in d x_{2} \mid W^{+}\left(t_{1}\right)=x_{1}\right)} \\
& \quad=\frac{\mathbf{P}\left(W\left(t_{2}\right) \in d x_{2}, W \in A, \inf _{t \in\left[t_{1}, t_{2}\right]} W(t) \geqslant 0 \mid W\left(t_{1}\right)=x_{1}\right)}{\mathbf{P}\left(W\left(t_{2}\right) \in d x_{2}, \inf _{t \in\left[t_{1}, t_{2}\right]} W(t) \geqslant 0 \mid W\left(t_{1}\right)=x_{1}\right)},
\end{aligned}
$$

но это и означает утверждение леммы. 
Лемма 3. Для любых $t_{1}, t_{2} \in(0,1]\left(t_{1}<t_{2}\right)$, положительных $x_{1}, x_{2}$ и $y \geqslant x_{1} \vee x_{2}$ справедливь равенства

$$
\begin{array}{r}
\mathbf{P}\left(W^{+}\left(t_{2}\right) \in d x_{2}, \sup _{t \in\left[t_{1}, t_{2}\right]} W^{+}(t) \leqslant y \mid W^{+}\left(t_{1}\right)=x_{1}\right) \\
=\frac{N_{1-t_{2}}\left(0, x_{2}\right)}{N_{1-t_{1}}\left(0, x_{1}\right)} \sum_{k=-\infty}^{+\infty} g\left(t_{2}-t_{1}, x_{1}, 2 k y+x_{2}\right) d x_{2}
\end{array}
$$

$u$

$$
\begin{aligned}
\mathbf{P}( & \left.W^{+}\left(t_{2}\right) \in d x_{2}, \sup _{t \in\left[t_{1}, t_{2}\right]} W^{+}(t)>y \mid W^{+}\left(t_{1}\right)=x_{1}\right) \\
& =\frac{N_{1-t_{2}}\left(0, x_{2}\right)}{N_{1-t_{1}}\left(0, x_{1}\right)} \sum_{k \in \mathbf{Z} \backslash\{0\}} g\left(t_{2}-t_{1},-x_{1}, 2 k y+x_{2}\right) d x_{2} .
\end{aligned}
$$

Д о к а з а т ель с т в о. Очевидно, что

$$
\begin{aligned}
& \mathbf{P}\left(W^{+}\left(t_{2}\right) \in d x_{2}, \sup _{t \in\left[t_{1}, t_{2}\right]} W^{+}(t) \leqslant y \mid W^{+}\left(t_{1}\right)=x_{1}\right) \\
&= \mathbf{P}\left(\sup _{t \in\left[t_{1}, t_{2}\right]} W^{+}(t) \leqslant y \mid W^{+}\left(t_{1}\right)=x_{1}, W^{+}\left(t_{2}\right)=x_{2}\right) \\
& \quad \times p^{+}\left(t_{1}, x_{1} ; t_{2}, x_{2}\right) d x_{2} .
\end{aligned}
$$

По лемме 2

$$
\begin{aligned}
\mathbf{P} & \left(\sup _{t \in\left[t_{1}, t_{2}\right]} W^{+}(t) \leqslant y \mid W^{+}\left(t_{1}\right)=x_{1}, W^{+}\left(t_{2}\right)=x_{2}\right) \\
= & \mathbf{P}\left(\sup _{t \in\left[t_{1}, t_{2}\right]} W(t) \leqslant y \mid W\left(t_{1}\right)=x_{1}, W\left(t_{2}\right)=x_{2}, \inf _{t \in\left[t_{1}, t_{2}\right]} \inf W(t) \geqslant 0\right) \\
= & \mathbf{P}\left(0 \leqslant \inf _{t \in\left[t_{1}, t_{2}\right]} W(t) \leqslant \sup _{t \in\left[t_{1}, t_{2}\right]} W(t) \leqslant y, W\left(t_{2}\right) \in d x_{2} \mid W\left(t_{1}\right)=x_{1}\right) \\
& \times\left[\mathbf{P}\left(\inf _{t \in\left[t_{1}, t_{2}\right]} W(t) \geqslant 0, W\left(t_{2}\right) \in d x_{2} \mid W\left(t_{1}\right)=x_{1}\right)\right]^{-1} .
\end{aligned}
$$

Совместное распределение минимума, максимума и положения в последний момент броуновского движения найдено, например, в $[5, \S 11]$. Исходя из этого распределения, нетрудно показать, что

$$
\begin{gathered}
\mathbf{P}\left(0 \leqslant \inf _{t \in\left[t_{1}, t_{2}\right]} W(t) \leqslant \sup _{t \in\left[t_{1}, t_{2}\right]} W(t) \leqslant y, W\left(t_{2}\right) \in d x_{2} \mid W\left(t_{1}\right)=x_{1}\right) \\
=\sum_{k=-\infty}^{+\infty} g\left(t_{2}-t_{1}, x_{1}, 2 k y+x_{2}\right) d x_{2}
\end{gathered}
$$

и

$$
\mathbf{P}\left(\inf _{t \in\left[t_{1}, t_{2}\right]} W(t) \geqslant 0, W\left(t_{2}\right) \in d x_{2} \mid W\left(t_{1}\right)=x_{1}\right)=g\left(t_{2}-t_{1}, x_{1}, x_{2}\right) d x_{2} .
$$


Из соотношений (35)-(38) следует, что

$$
\begin{aligned}
\mathbf{P}( & \left.W^{+}\left(t_{2}\right) \in d x_{2}, \sup _{t \in\left[t_{1}, t_{2}\right]} W^{+}(t) \leqslant y \mid W^{+}\left(t_{1}\right)=x_{1}\right) \\
& =\frac{p^{+}\left(t_{1}, x_{1} ; t_{2}, x_{2}\right)}{g\left(t_{2}-t_{1}, x_{1}, x_{2}\right)} \sum_{k=-\infty}^{+\infty} g\left(t_{2}-t_{1}, x_{1}, 2 k y+x_{2}\right) d x_{2} .
\end{aligned}
$$

Откуда, учитывая соотношение (21), получаем (33). Далее, сумма левых частей соотношений $(33)$ и $(34)$ равна $p^{+}\left(t_{1}, x_{1} ; t_{2}, x_{2}\right) d x_{2}$, поэтому, снова применяя (21) и доказанное соотношение (33), получаем (34). Лемма доказана.

Возвратимся к соотношению (22). По лемме 3 при $i>0, x_{i} \in(0,1]$, $x_{i+1} \in(0,1]$ выполнено равенство

$$
\begin{aligned}
\mathbf{P}^{\left(t_{i}, x_{i}\right)} & \left(W^{+}\left(\frac{t_{i+1}}{t_{m}}\right) \in \frac{d x_{i+1}}{\sqrt{t_{m}}}, \sup _{t \in\left[t_{i}, t_{i+1}\right]} W^{+}\left(\frac{t}{t_{m}}\right) \leqslant \frac{1}{\sqrt{t_{m}}}\right) \\
= & \frac{N_{1-t_{i+1} / t_{m}}\left(0, x_{i+1} / \sqrt{t_{m}}\right)}{N_{1-t_{i} / t_{m}}\left(0, x_{i} / \sqrt{t_{m}}\right)} \sum_{k=-\infty}^{+\infty} g\left(t_{i+1}-t_{i}, x_{i}, 2 k+x_{i+1}\right) d x_{i+1}
\end{aligned}
$$

откуда, учитывая соотношение (21), находим, что при $i>0, x_{i} \in(0,1]$

$$
\begin{aligned}
& \mathbf{P}^{\left(t_{j}, x_{j}\right)}\left(W^{+}\left(\frac{t_{j+1}}{t_{m}}\right) \in \frac{d x_{j+1}}{\sqrt{t_{m}}}, \sup _{t \in\left[t_{j}, t_{j+1}\right]} W^{+}\left(\frac{t}{t_{m}}\right)>\frac{1}{\sqrt{t_{m}}}\right) \\
& =\frac{N_{1-t_{j+1} / t_{m}}\left(0, x_{j+1} / \sqrt{t_{m}}\right)}{N_{1-t_{j} / t_{m}}\left(0, x_{j} / \sqrt{t_{m}}\right)} d x_{i+1} \\
& \quad \times \begin{cases}\sum_{k \in \mathbf{Z} \backslash\{0\}} g\left(t_{i+1}-t_{i},-x_{j}, 2 k+x_{j+1}\right), & x_{j+1} \in(0,1], \\
\frac{1}{\sqrt{t_{m}}}\left(\frac{t_{i+1}-t_{i}}{t_{m}}, \frac{x_{j}}{\sqrt{t_{m}}}, \frac{x_{j+1}}{\sqrt{t_{m}}}\right), & x_{j+1}>1 .\end{cases}
\end{aligned}
$$

Заменяя в соотношении $(22)$ все вероятности в правой части в соответствии с формулами $(23),(25),(26),(39)$ и (40) и замечая, что

$$
\begin{aligned}
& N_{1-t_{1} / t_{m}}\left(0, \frac{x_{1}}{\sqrt{t_{m}}}\right) \prod_{i=1}^{m-1} \frac{N_{1-t_{i+1} / t_{m}}\left(0, x_{i+1} / \sqrt{t_{m}}\right)}{N_{1-t_{i} / t_{m}}\left(0, x_{i} / \sqrt{t_{m}}\right)}=\frac{1}{2}, \\
& \frac{1}{\sqrt{t_{m}}} g\left(\frac{t_{i+1}-t_{i}}{t_{m}}, \frac{x_{i}}{\sqrt{t_{m}}}, \frac{x_{i+1}}{\sqrt{t_{m}}}\right)=g\left(t_{i+1}-t_{i}, x_{i}, x_{i+1}\right),
\end{aligned}
$$

получаем, что при $j=0,1, \ldots, m-1$

$$
\begin{aligned}
P_{j}= & \sqrt{\frac{\pi t_{m}}{2}} \int_{G_{j}\left(a_{1}, \ldots, a_{m}\right)} \ldots \prod_{i=0}^{j-1} p_{1}\left(t_{i}, x_{i} ; t_{i+1}, x_{i+1}\right) p_{2}\left(t_{j}, x_{j} ; t_{j+1}, x_{j+1}\right) \\
& \times \prod_{i=j+1}^{m-1} g\left(t_{i+1}-t_{i}, x_{i}, x_{i+1}\right) d x_{1} \cdots d x_{m}
\end{aligned}
$$


Осталось найти вероятность $P_{m}$. Вместо $(22)$ получаем формулу

$$
\begin{aligned}
P_{m}= & \int \ldots \int \prod_{G_{m}\left(a_{1}, \ldots, a_{m}\right)} \prod_{i=0}^{m-1} \mathbf{P}^{\left(t_{i}, x_{i}\right)}\left(W^{+}\left(\frac{t_{i+1}}{t_{m}}\right) \in \frac{d x_{i+1}}{\sqrt{t_{m}}}, \sup _{t \in\left[t_{i}, t_{i+1}\right]} W^{+}\left(\frac{t}{t_{m}}\right) \leqslant \frac{1}{\sqrt{t_{m}}}\right) \\
& \times \mathbf{P}^{\left(t_{m}, x_{m}\right)}\left(\sup _{t \in\left[t_{m},+\infty\right)} W^{+}\left(\frac{t}{t_{m}}\right)>\frac{1}{\sqrt{t_{m}}}\right) .
\end{aligned}
$$

Но последняя вероятность равна (вспомним определение процесса $W^{+}(t)$ при $t \geqslant 1$ )

$$
\mathbf{P}\left(\sup _{t \in[1, \tau]} W(t)>\frac{1}{\sqrt{t_{m}}} \mid W(1)=\frac{x_{m}}{\sqrt{t_{m}}}\right)=x_{m},
$$

причем последнее равенство является аналогом формулы для вероятности разорения (см. [8, гл. 14]). Следовательно, ввиду соотношений (25), (39) и (41) справедливо равенство

$$
P_{m}=\sqrt{\frac{\pi t_{m}}{2}} \int_{G_{m}\left(a_{1}, \ldots, a_{m}\right)} \ldots \prod_{i=0}^{m-1} p_{1}\left(t_{i}, x_{i} ; t_{i+1}, x_{i+1}\right) x_{m} d x_{1} \cdots d x_{m} .
$$

Подставляя выражения для вероятностей $P_{j}$ из формул (43) и (44) в соотношение (19) и учитывая (18), получаем утверждение теоремы 2.

В качестве дополнения к теореме 2 отметим (см. соотношение (18)), что если хотя бы одно из чисел $a_{1}, \ldots, a_{m}$ больше или равно 1 , то

$$
\begin{aligned}
\mathbf{P} & \left(Y\left(t_{i}\right)>a_{i}, i=1, \ldots, m\right) \\
& =\sqrt{\frac{2}{\pi t_{m}}} \mathbf{P}\left(W^{+}\left(\frac{t_{i}}{t_{m}}\right)>\frac{a_{i}}{\sqrt{t_{m}}}, i=1, \ldots, m\right) \\
& =\sqrt{\frac{2}{\pi t_{m}}} \int_{G_{0}\left(a_{1}, \ldots, a_{m}\right)} \ldots \int \prod_{i=0}^{m-1} p^{+}\left(\frac{t_{i}}{t_{m}}, \frac{x_{i}}{\sqrt{t_{m}}} ; \frac{t_{i+1}}{t_{m}}, \frac{x_{i+1}}{\sqrt{t_{m}}}\right) \frac{1}{\left(\sqrt{t_{m}}\right)^{m}} d x_{1} \cdots d x_{m},
\end{aligned}
$$

Откуда, замечая, что (см. соотношения (20), (21), (41) и (42))

$$
\begin{aligned}
\prod_{i=0}^{m-1} & p^{+} \\
\quad & \left(\frac{t_{i}}{t_{m}}, \frac{x_{i}}{\sqrt{t_{m}}} ; \frac{t_{i+1}}{t_{m}}, \frac{x_{i+1}}{\sqrt{t_{m}}}\right) \\
& \left.=\sqrt{\frac{\pi}{2}}, \frac{x_{1}}{\sqrt{t_{m}}}\right) \prod_{i=1}^{m-1} g\left(\frac{t_{i+1}-t_{i}}{t_{m}}, \frac{x_{i}}{\sqrt{t_{m}}}, \frac{x_{i+1}}{\sqrt{t_{m}}}\right)\left(\sqrt{t_{m}}\right)^{m+1} \prod_{i=1}^{m-1} g\left(t_{i+1}-t_{i}, x_{i}, x_{i+1}\right),
\end{aligned}
$$

получаем равенство

$$
\begin{aligned}
& \mathbf{P}\left(Y\left(t_{i}\right)>a_{i}, i=1, \ldots, m\right) \\
& \quad=\int_{G_{0}\left(a_{1}, \ldots, a_{m}\right)} \ldots \int_{1} f\left(t_{1}, x_{1}\right) \prod_{i=1}^{m-1} g\left(t_{i+1}-t_{i}, x_{i}, x_{i+1}\right) d x_{1} \cdots d x_{m} .
\end{aligned}
$$


Далее, поскольку состояние 0 является поглощающим для процесса $Y$, то помимо рассмотренных в теореме 2 вероятностей разумно рассмотреть при положительных $a_{1}, \ldots, a_{m}$

$$
\mathbf{P}\left(Y\left(t_{i}\right)>a_{i}, i=1, \ldots, m-1 ; Y\left(t_{m}\right)=0\right) .
$$

Очевидно, что эта вероятность может быть вычислена по формуле

$\mathbf{P}\left(Y\left(t_{i}\right)>a_{i}, i=1, \ldots, m-1\right)-\mathbf{P}\left(Y\left(t_{i}\right)>a_{i}, i=1, \ldots, m-1 ; Y\left(t_{m}\right)>0\right)$, учитывая, что две последние вероятности находятся по теореме 2.

\section{СПИСОК ЛИТЕРАТУРЫ}

1. Afanasyev V.I., Geiger J., Kersting G., Vatutin V.A. Criticality for branching processes in random environment. - Ann. Probab., 2005, v. 33, № 2, p. 645-673.

2. Афанасьев В. И. Закон арксинуса для ветвящихся процессов в случайной среде и процессов Гальтона-Ватсона. - Теория вероятн. и ее примен., 2006, т. 51, в. 3, c. $449-464$.

3. Афанасьев В. И. О моменте достижения фиксированного уровня критическим ветвящимся процессом в случайной среде. - Дискретн. матем., 1999, т. 11, № 4, c. $33-47$.

4. Durrett R. T., Iglehart D. L. Functionals of Brownian meander and Brownian excursion. - Ann. Probab., 1977, v. 5, № 1, p. 130-135.

5. Биллингсли П. Сходимость вероятностных мер. М.: Наука, 1977, 352 с.

6. Iglehart D. L. Functional central limit theorems for random walks conditioned to stay positive. - Ann. Probab., 1974, v. 2, № 4, p. 608-619.

7. Bolthausen E. On a functional central limit theorem for random walks conditioned to stay positive. - Ann. Probab., 1976, v. 4, № 3, p. 480-485.

8. Феллер В. Введение в теорию вероятностей и ее приложения. Т. 1. М.: Мир, 1984, $528 \mathrm{c}$.

9. Феллер В. Введение в теорию вероятностей и ее приложения. Т. 2. М.: Мир, 1984, $752 \mathrm{c}$. 